\title{
Dysfunction of D2 dopamine receptor expressing neurons underlies manic-like behaviors in mice modeling SHANK3 duplication disorder
}

Jimmy Holder ( $\boldsymbol{\nabla}$ holder@bcm.edu )

Baylor College of Medicine https://orcid.org/0000-0001-5595-0458

Kaifang Pang

Baylor College of Medicine

Michel Weiwer

Broad Institute

Kihoon Han

Korea University

Wei Wang

Baylor College of Medicine

Theresa Estiphan

University of Michigan

Blake Vuocolo

Baylor College of Medicine

Karen Jaunarajs

University of Alabama at Birmingham

Edward Holson

Amathus Therapeutics

Mike Lewis

Sage Therapeutics (United States)

Qihong Xu

Broad Institute

Jennifer Gale

Broad Institute

Steen Pedersen

Ross University of Medicine

\section{Robia Pautler}

Baylor College of Medicine

\section{David Standaert}

University of Alabama at Birmingham https://orcid.org/0000-0003-2921-8348

Florence Wagner 
Broad Institute

\section{Zhandong Liu}

Baylor College of Medicine

\section{Huda Zoghbi}

Baylor College of Medicine https://orcid.org/0000-0002-0700-3349

\section{Article}

Keywords: SHANK3, neuropsychiatric disorders, genetics

Posted Date: July 12th, 2021

DOI: https://doi.org/10.21203/rs.3.rs-646721/v1

License: (c) (1) This work is licensed under a Creative Commons Attribution 4.0 International License. Read Full License 


\section{Abstract}

While the contributions of some genes to neuropsychiatric disorders are clear, the downstream neuronal effects are poorly understood. Over-expression of SHANK3, which encodes a key synaptic protein, causes neuropsychiatric phenotypes in humans and manic-like behavior in mice providing an opportunity to interrogate the role of SHANK3 in a subset of neurons that might underlie the manic-like behavior. Herein, we describe Shank3's critical role in D2 dopamine receptor (D2dr) neurons and show that Shank3 overexpression causes increased synaptic neurotransmission in D2dr, but not D1dr, expressing striatal medium spiny neurons. Either pharmacologic D2dr inhibition or genetic normalization of Shank3 abundance in D2-neurons ameliorates manic-like behaviors. Integrating bioinformatic analyses of Shank3's striatal interactome, D1 and D2 dopamine receptor binding proteins, and single-cell RNA-seq datasets, we demonstrate a functional relationship between Shank3 and the D2dr-but not the D1dr. Thus, while Shank3 is over-expressed in both D1 and D2 dopamine receptor expressing striatal neurons, D2 neuronal dysfunction causes manic-like behaviors.

\section{Introduction}

Neuropsychiatric disorders have strong genetic underpinnings. For example, the heritability for schizophrenia, bipolar disorder and autism are all estimated to be greater than $80 \%^{1,2}$. For some individuals diagnosed with neuropsychiatric disorders, highly penetrant variants in a single gene drive phenotypes. This has been most clearly demonstrated in autism spectrum disorders (ASDs), where more than 100 genes have been linked to ASD etiology ${ }^{3}$. It has been more difficult, however, to identify which single genetic lesions drive other neuropsychiatric disorders' clinical expression, like bipolar disorder. In the rare cases in which such a genetic aberration is identified, using model experimental systems to investigate the pathophysiology can help identify the underlying mechanisms driving neuropsychiatric phenotypes and, potentially, lead to rationally designed therapies.

We have previously reported that one such monogenic abnormality leads to neuropsychiatric phenotypes due to SHANK3 overexpression ${ }^{4}$. Individuals with duplications encompassing SHANK3 have been diagnosed with bipolar disorder, atypical Attention Deficit Hyperactivity Disorder and other behavioral issues $^{4-6}$. Moreover, we reported that mice with a Shank3 genomic duplication express manic-like phenotypes that respond to valproate but resist lithium ${ }^{4}$. While this work elucidated behavioral and molecular abnormalities associated with Shank3 overexpression, an interesting unanswered question is how dysfunction in various brain regions and neuronal subtypes contributes to the neuropsychiatric phenotypes associated with Shank3 overexpression.

Here, we investigated the striatum's role in behavioral abnormalities associated with Shank3 overexpression. We chose to investigate the role of the striatum in the phenotypes associated with SHANK3 Duplication syndrome for the following reasons: 1 . Shank3 is the most abundantly expressed Shank paralog in the striatum ${ }^{7} 2$. anti-psychotic medications, which are commonly used for the treatment of manic episodes ${ }^{8}$, inhibit D2 dopamine receptors which are most abundantly expressed in the striatum 
and 3. we previously discovered an enhanced effect of amphetamine in Shank3 over-expressing mice 4 which suggests dysfunction of the dopamine signaling system. We discovered that dysfunction in D2 dopamine receptor expressing neurons drove select manic-like behaviors in mice and rescued these behaviors both genetically and pharmacologically. We used bioinformatic analyses combining a striatal specific Shank3 interactome with single-cell RNA-sequencing data to converge on a network of Shank3 interacting proteins that functionally interact with D2 dopamine receptor interacting proteins and coexpressed genes. These data demonstrate a critical role for Shank3 in the normal function of D2dr expressing neurons and molecular interdependency of the Shank3 interactome with the D2 dopamine receptor.

\section{Results}

\section{D2 dopamine receptor medium spiny neuron dysfunction}

We previously discovered that CA1 pyramidal hippocampal neurons in Shank3 overexpressing (Shank3 TG) mice have increased synaptic neurotransmission ${ }^{4}$; however, it is unknown if other neuronal subtypes in Shank3 TG mice have synaptic dysfunction. Brain regions and neurons that are potentially more relevant to manic-like behavior, like the striatum ${ }^{9-11}$ where Shank3 expression is very high compared to its paralogs, have not been evaluated previously. To fill this knowledge gap, we evaluated Shank3 TG mice for changes in striatal medium-spiny neurons' neurophysiological properties by patch-clamp electrophysiology (Fig. 1A). We found that Shank3 TG mice had more frequent (Fig. 1B), but normal amplitude (Fig. 1C), miniature excitatory post-synaptic currents (mEPSCs) compared with controls, demonstrating increased functional synapse number with normal strength. To help pinpoint which neurons drove this increased synaptic neurotransmission, we labeled D2 dopamine receptor expressing neurons with TdTomato using the Ai9 mouse-line that expresses TdTomato in response to Cre-mediated recombination at the Rosa26 locus ${ }^{12}$. We again measured mEPSCs via patch-clamp electrophysiology in D2 (TdTomato positive) and D1 (unlabeled) medium-spiny neurons (MSNs). D2 MSNs demonstrated elevated mEPSC frequency (Fig. 1E) but normal amplitude (Fig. 1F). In contrast, D1 MSNs had normal mEPSC frequency and amplitude. Together, these data demonstrate an increased number of functional excitatory synapses in D2 dopamine receptor expressing neurons of Shank3 TG mice.

\section{Structural analysis of the striatum}

To further investigate the striatal phenotypes in Shank3 TG mice, we assessed the striatum's structure from the macro- to nanoscale. For the macrostructure assessment, we used Magnetic Resonance Imaging (MRI) to determine the volume and connectivity via Diffusion Tensor Imaging (DTI) (Fig. 2A); we found no significant differences in either the volume (Fig. 2B) or the fractional anisotropy (FA) (Fig. 2C) of the caudate of Shank3 TG mice compared with wild-type littermates. Further examination of the MRI data for changes in volume of specific brain structures identified little difference in the Shank3 TG mice (Supplemental Table I). We identified no significant differences in fractional anisotropy in any brain region analyzed (Supplemental Table II). 
Our previous examination of cultured Shank3 overexpressing hippocampal neurons revealed increased excitatory synapse numbers ${ }^{4}$. The impact of Shank3 overexpression on other brain regions, however, has not been evaluated. We used Golgi staining to examine neuronal morphology (Fig. 2D) and evaluate changes in medium spiny neurons' dendritic spine density. Total spine density was significantly higher in the Shank3 TG mice striata compared with control animals (Fig. 2E). We also used transmission electron microscopy to perform ultrastructural evaluation and examine synaptic structure (Fig. 2F). The length (Fig. 2G) and thickness (Fig. 2H) of the post-synaptic densities (PSDs) were measured, and we found no significant differences in either when compared with controls. These data corroborate the neurophysiologic data demonstrating that increased synapse number drives D2 dopamine receptor expressing MSN's increased synaptic neurotransmission.

Dopamine content upon SHANK3 overexpression

Elevated synaptic dopamine content is known to induce hyperactivity. For example, dopamine transporter deficient (DAT) mice, a model of manic-like behavior, have elevated synaptic dopamine content ${ }^{13,14}$. Therefore, we investigated whether Shank3 overexpression causes changes in striatal synaptic dopamine. We measured extracellular dopamine (DA) concentration via in vivo microdialysis using high performance liquid chromatography both at baseline and in response to amphetamine ( $2 \mathrm{mg} / \mathrm{kg} / \mathrm{dose}$, ip), which is known to increase dopamine concentration at the synaptic cleft. The baseline synaptic dopamine concentration was unaltered in Shank3 TG mice compared with their wild-type littermates (Fig. 2I). Both genotypes had robust rises in synaptic dopamine levels in response to intraperitoneal amphetamine injections (Fig. $2 \mathrm{I}$ and $2 \mathrm{~J}$ ). However, we found no significant difference in DA content in Shank3 TG mice except for a slight reduction in DA 20 minutes after amphetamine injection versus wildtype littermates (Fig. 2J). Therefore, we conclude that changes in dopamine efflux do not drive the maniclike behaviors of Shank3 overexpressing mice.

\section{Rescue of select manic-like behaviors by D2 dopamine receptor antagonists}

D2 dopamine receptor antagonists are used clinically to treat some individuals with mania ${ }^{8}$. Given the altered synaptic properties of D2dr expressing neurons in our Shank3 overexpressing mice, we hypothesized that D2 antagonists would improve their manic-like phenotypes. We utilized two novel D2 dopamine receptor antagonists, BRD4018 and BRD5814, biased toward inhibition of downstream $\beta$ arrestin and away from small G-protein inhibition (Fig. 3A $)^{15}$. $\beta$-arrestin biased D2dr antagonists are considered promising antipsychotics because of their ability to rescue a rodent model of psychosis amphetamine-induced hyperactivity $(\mathrm{AlH})$ without unwanted motoric effects (reduced latency on rotarod $)^{15-17}$. It is hypothesized that this specificity of $\beta$-arrestin antagonism without significantly impacting G-protein function reduces side-effects associated with clinically available anti-psychotics.

We tested these D2 dopamine receptor antagonists' efficacy against manic-like behaviors in Shank3 TG mice by injecting BRD4018, BRD5814 or vehicle intraperitoneally with a sub-acute three injection paradigm of $10 \mathrm{mg} / \mathrm{kg}$ (BRD4018) or $10 \mathrm{mg} / \mathrm{kg}$ (BRD5814) giving the final injection one hour prior to each behavioral analysis (Fig. 3B). These doses were chosen as they result in $>50 \%$ D2dr occupancy based 
upon PET displacement of radiolabeled Raclopride ${ }^{15}$. We measured total activity and rearing in a novel environment at baseline and post-amphetamine injection and performed acoustic startle response (ASR) with prepulse inhibition (PPI) and tail suspension testing to evaluate for behavioral rescue. Both BRD4018 and BRD5814 rescued baseline hyperactivity in Shank3 TG mice (Fig. 3C and G). BRD4018 completely blocked exaggerated amphetamine induced hyperactivity in Shank3 TG mice (Fig. 3D). BRD 5814 partially rescued the exaggerated amphetamine-induced hyperactivity in Shank3 TG mice (Fig. 3H). As with total activity, both BRD4018 (Fig. 3E and F) and BRD5814 (Fig. 3I and J) rescued increased rearing in Shank3 TG mice both at baseline and in response to amphetamine. In contrast to motor behaviors, no consistent rescue of abnormalities in maximal acoustic startle response or immobility in tail suspension testing of Shank3 TG mice were observed (Supplemental Figs. 1 and 2). Thus, D2dr dopamine receptor antagonists rescue select manic-like behaviors in Shank3 overexpressing mice.

\section{Rescue of select manic-like behaviors by normalizing Shank3 in D2 dopamine receptor expressing neurons.}

To confirm D2 dopamine receptor expressing neurons play a critical role in Shank3 overexpression's motor manifestations, we endeavored to normalize Shank3 abundance in these neurons. We created new conditional Shank3 overexpressing mice using BAC transgenesis (Fig. 4A), the same method we used to create the original Shank3 TG line and added LoxP sites that we introduced upstream and downstream of the Shank3 coding region (Shank3 Flox TG). The Shank3 Flox TG mice express the EGFP-tagged Shank3 protein at the expected molecular size although at approximately $75 \%$ the abundance of the original Shank3 TG allele (Fig. 4B) and approximately 35\% overexpression of Shank3. We confirmed this conditional allele's validity by mating these mice with Nes-Cre expressing mice and western blotting their cerebral tissue to confirm reduced transgene expression (Fig. 4C). We mated the Shank3 Flox TG mice with D2-Cre mice to normalize Shank3 abundance in D2 dopamine receptor-expressing neurons (Shank3 Flox TG/D2-Cre) and then assessed for behavior (Fig. 4D). At baseline, there was no significant difference in total locomotor activity (Fig. 4E) and a slight reduction in rearing (Fig. 4G) in the open field test compared with wild-type littermates. When we administered amphetamine, the Shank3 Flox TG mice had an exaggerated increase in locomotor activity similar to that seen in the original Shank3 overexpressing mice (Fig. 4F). In mice with normalized Shank3 expression in D2 dopamine receptor expressing neurons (Shank3 Flox TG/D2-Cre), the total locomotor response to amphetamine was similar to that in wild-type littermates (Fig. 4F). Similarly, normalizing Shank3 in D2dr neurons resulted in a blunted rearing response to amphetamine below that of wild-type mice (Fig. 4H). Together, these data confirm critical role of D2 dopamine receptor expressing neurons in the exaggerated amphetamine-induced hyperactivity with Shank3 overexpression.

In contrast to the original Shank3 TG mice, Shank3 Flox TG mice did not have a significant difference in acoustic startle response, likely due to Shank3's modestly elevated expression in this line (Supplemental Fig. 3). However, Shank3 Flox TG mice did manifest a reduced immobility time in the tail suspension test similar to that of the original Shank3 TG mice which was not rescued by normalizing Shank3 abundance 
in D2dr expressing neurons (Supplemental Fig. 3). Together, these data confirm that D2 dopamine receptor expressing neuronal dysfunction underlies exaggerated motor response to amphetamine but not other manic-like behaviors due to Shank3 overexpression.

\section{D2 dopamine receptor antagonists improve symptoms in individuals with SHANK3 duplications.}

The second patient we described in our original report on SHANK3 duplication syndrome was a 35 year old man with epilepsy and bipolar disorder ${ }^{4}$. His detailed medical history indicated that, while he had used many medications to manage his mood disorder over many years, aripiprazole, a newer atypical antipsychotic with antagonistic activity at post-synaptic D2 dopamine receptors, improved his mood symptoms.

We identified a new patient with a 22q13 duplication encompassing SHANK3. She was originally evaluated at age 7 years due to inattentiveness and behavioral concerns, and was diagnosed with Attention Deficit Hyperactivity Disorder. She was initially treated with methylphenidate which resulted in significant behavioral decline including aggression and poor mood. When her impulsivity significantly increased and aggression continued to worsen, she was prescribed risperidone, an atypical antipsychotic with D2dr inhibitory activity. This led to improvement in her behavior.

A third patient with SHANK3 duplication syndrome was diagnosed with bipolar disorder, Tourette syndrome and obsessive compulsive disorder $(O C D)^{5}$. This individual also reportedly responded favorably to aripiprazole. Together, these data demonstrate that antagonizing the D2 dopamine receptor also provides symptomatic relief in individuals with neuropsychiatric diagnoses stemming from SHANK3 overexpression.

\section{Functional interactions between the Shank3-protein interaction network and D1 and D2 dopamine receptors.}

To identify which molecular abnormalities drive changes in D2dr neurons and subsequent manic-like behaviors in Shank3 overexpressing mice, we utilized proteomics to identify proteins that interact with Shank3 specifically in the striatum. We dissected striata from Shank3 TG or wild-type mice, isolated synaptosomes and then immunoprecipitated EGFP-Shank3. The proteins that bound specifically to EGFPShank3 were identified by mass spectrometry. In total, 451 proteins were identified within the Shank3 interactome (Supplemental Table III). We further overlapped these proteins with Shank3 interactors we previously identified using yeast two-hybrid screening ${ }^{18}$ and in vivo immunoprecipitation ${ }^{4}$ to obtain 84 high-confidence Shank3 interactors (Supplemental Table IV).

To discover the functional relationship between Shank3 interactors and D2/D1 dopamine receptors, we first built a protein interaction network using the BioGRID database ${ }^{19}$ (Methods). We then extracted a protein interaction subnetwork consisting of high-confidence Shank3 interactors, D2dr with its interactors (Supplemental Table V), and D1 dr with its interactors (Supplemental Table VI) (Fig. 5A). We found that high-confidence Shank3 interactors are more densely connected with D2dr and its interactors than D1dr 
and its interactors (Fig. 5A), suggesting that Shank3 interactors function more closely with D2dr than D1dr. Further, shortest path length analysis revealed that Shank3 interactors have significantly shorter path lengths to D2dr than to D1dr (Fig. 5B), with the median path between Shank3 interactors and D2dr being two and that between Shank3 interactors and D1dr being three. Together, these data reveal a significantly closer physical interaction between high-confidence Shank3 interactors and the D2dr than the D1dr and thus closer functional connection.

Co-expression analysis of disease genes using single-cell expression data can inform whether the disease genes tend to function together at the single-cell level ${ }^{20}$. We next constructed a gene coexpression network consisting of high-confidence Shank3 interactors and D2dr in D2-expressing neurons (D2 neurons) (Fig. 5C) and a gene co-expression network consisting of Shank3 interactors and D1dr in D1-expressing neurons (D1 neurons) (Fig. 5D) using single-cell RNA sequencing data of mouse striatum

21 (Methods). We found that high-confidence Shank3 interactors are densely co-expressed with D2dr in D2 neurons (Fig. 5C) whereas few of the Shank3 interactors are co-expressed with D1dr in D1 neurons (Fig. 5D). We also calculated the Spearman's correlation of Shank3 interactors with D2dr in D2 neurons (Fig. 5E) and that of Shank3 interactors with D1dr in D1 neurons (Fig. 5F). We observed that Shank3 interactors show significant positive correlation with D2dr in D2 neurons (Fig. 5E) whereas the correlation between Shank3 interactors and D1dr tends to be negative (Fig. 5F). Together, these data demonstrated that Shank3 interactors are more likely to share functions with D2dr in D2 neurons than D1dr in D1 neurons.

Furthermore, we calculated the Spearman's correlation with Shank3 interactors for all genes expressed in D2/D1 neurons. We found that D2dr is among the top genes that positively correlate with Shank3 interactors in D2 neurons (Fig. 5G), whereas D1dr is among the bottom genes that positively correlate with Shank3 interactors in D1 neurons (Fig. 5H). This result reveals a highly close functional relationship between D2dr and Shank3 interactors in D2 neurons. Collectively, our protein interaction network and coexpression analyses both demonstrate a close functional relationship between the striatal Shank3 interactome and the D2 dopamine receptor that is absent for the D1 dopamine receptor.

\section{Discussion}

Bipolar disorder is a mood disorder characterized by shifts between depressed and manic states. Despite having a significant genetic basis, how genetic variation contributes to the bipolar disorder's pathophysiology is unclear. Much of the current work being done to understand the genetic basis of psychiatric disorders depends upon identifying common variations that each contribute to a small increase in disease development risk ${ }^{22}$. However, because the individual contributions of these variants to psychiatric disease pathophysiology are so small, pinpointing their molecular and neurophysiologic consequences is challenging.

Here, we focused on one rare but highly penetrant genomic abnormality-SHANK3 duplication, which is associated with psychiatric phenotypes including bipolar disorder-and its pathophysiologic 
consequences. We previously reported that mice modeling a SHANK3 duplication have multiple behavioral abnormalities that mimic manic-like behavior ${ }^{4}$.

In this study, we discovered that striatal dysfunction drives select manic-like behavior in mice. We identified increased synaptic neurotransmission in D2, but not D1, dopamine receptor expressing medium spiny neurons in Shank3 overexpressing mice. This is associated with increased dendritic spine number within the striatum. Intriguingly, genetically depleted Shank3 mice (Shank3 KO) have also been reported to have neurophysiologic dysfunction in D2 but not D1 dopamine receptor expressing neurons within the striatum and reductions in both mEPSC frequency and amplitude ${ }^{23}$. Thus, D2 dopamine receptor expressing neurons are exquisitely sensitive to the dose of Shank3 while D1 neurons are not.

Normalizing Shank3 in D2 dopamine receptor expressing neurons rescued enhanced sensitivity to amphetamine induced hyperactivity without altering other Shank3 overexpression behavioral abnormalities. Moreover, inhibiting D2 dopamine receptors with small molecule antagonists can similarly rescue enhanced amphetamine induced hyperactivity without improving other behavioral abnormalities increased acoustic startle response. Together these data demonstrate that D2 dopamine receptor expressing neuronal dysfunction drives select manic-like behaviors related to Shank3 overexpression.

D2 dopamine receptors are present both on the post-synaptic surface as well as presynaptic terminals where they can regulate dopamine release. However, using in vivo microdialysis with HPLC, we found no significant abnormality in either basal dopamine release or release in response to amphetamine. These data demonstrate that dysregulation of dopamine release does not drive abnormal behaviors in Shank3 TG mice.

To examine why Shank3 abundance in D2, but not D1, dopamine receptor-expressing striatal neurons result in these neurons' dysfunction, we identified the Shank3-protein interaction network in Shank3 overexpressing mice striata. Utilizing previously published single-cell RNA-seq data from mouse striata, we identified significant overlap between the Shank3-protein interaction and D2 (but not D1) dopamine receptor co-expression networks. We also found that Shank3-protein interactors are more closely physically linked to the D2dr than the D1dr interactors. Taken together, these data demonstrate Shank3 is functionally linked to a D2 dopamine receptor co-expressed protein complex but not the D1 dopamine receptor co-expressed protein network.

We next explored the clinical relevance of our discovery that D2 dopamine receptor inhibition improves phenotypes in Shank3 overexpressing mice. Antipsychotic medications are frequently prescribed for mood stabilization in individuals with bipolar disorder ${ }^{8}$; however, because we know so little about which genetic variants predispose to bipolar disorder, these medications' use is empirical. We did find that individuals harboring SHANK3 duplications have behavioral phenotypes that respond to antipsychotic medications confirming the pharmaco-genetic association between Shank3 overexpression and the D2 dopamine receptor antagonists identified in mice. Intriguingly, neuropsychiatric disorders due to mutations in genes encoding Shank3 striatal interacting proteins have been identified. For at least two of 
these, SYNGAP1 $1^{24,25}$ and $T A N C 2^{26}$, significant behavioral and psychiatric phenotypes have been reported. We speculate that dysfunction of striatal neurons, perhaps D2dr expressing neurons, underlie these phenotypes and warrants further investigation.

The pathophysiologic mechanisms leading to bipolar disorder are poorly understood. Hypotheses have included mitochondrial dysfunction, circadian rhythm abnormalities and excitatory/inhibitory imbalance ${ }^{8,27}$. Several lines of evidence suggest that an imbalance of excitation/inhibition leading to neuronal hyperexcitability underlie bipolar disorder. First, neurons derived from bipolar disorder patients' induced pluripotent stem cells (iPSCs) have a hyperexcitable phenotype $\mathrm{e}^{28}$ as measured by spontaneous action potentials during patch clamping. Second, multiple bipolar disorder patient neuroimaging studies have used magnetic resonance spectroscopy (MRS) to identify increased Glx (glutamine and glutamate peaks) by magnetic resonance spectroscopy (MRS) (reviewed in ${ }^{29}$ ). Finally, two medications commonly used to stabilize mood in bipolar disorder, valproic acid and lamotrigine, decrease neuronal excitation ${ }^{8}$. Despite these data, though, whether specific neuronal sub-types' dysfunction can drive manic behaviors was largely unknown. The data reported here using a genetically accurate animal model of neuropsychiatric disorders highlight the role of D2 receptor neurons and their enhanced excitability for mania-like behavior. Taken together, these data and future studies on additional proteins critical for D2 $r$ neuron function and excitability could provide better understanding of their potential contribution to bipolar disorder and its pathophysiology and to the development of rational therapies.

\section{Methods}

\section{Mouse husbandry}

All mice were maintained in a specific pathogen free facility with a constant light cycle (14 light: 10 hours dark). Shank3 TG ${ }^{4}$ and Shank3 Flox TG mice were made in house and maintained on an FVB background. D2-cre mice (B6.FVB(Cg)-Tg(Drd2-cre)ER44Gsat/Mmcd) were purchased from MMRRC and back-crossed for more than six generations to $\mathrm{C} 57 / \mathrm{bl6J}$ mice. All mice for behavioral analysis were male F1 hybrids of FVBXC57/bl6J. All procedures for the creation and use of these mice was approved by the Institutional Animal Care and Use Committee for Baylor College of Medicine.

\section{Slice electrophysiology}

Mice were anesthetized with isoflurane and coronal slices (350 $\mu \mathrm{m}$ thick) containing dorsal striatum were cut with a vibratome (Leica Microsystems Inc, Buffalo Grove, IL) in a chamber filled with chilled cutting solution containing (mM) 110 Choline-Chloride, $25 \mathrm{NaHCO}_{3}, 25$ D-glucose, 11.6 sodium ascorbate, 7 $\mathrm{MgSO}_{4}, 3.1$ sodium pyruvate, $2.5 \mathrm{KCl}, 1.25 \mathrm{NaH}_{2} \mathrm{PO}_{4}$ and $0.5 \mathrm{CaCl}_{2}$. The slices were then incubated in artificial cerebrospinal fluid (ACSF, in $\mathrm{mM}$ ) containing $119 \mathrm{NaCl}, 26.2 \mathrm{NaHCO}_{3}, 11 \mathrm{D}$-glucose, $3 \mathrm{KCl}, 2$ $\mathrm{CaCl}_{2}, 1 \mathrm{MgSO}_{4}, 1.25 \mathrm{NaH}_{2} \mathrm{PO}_{4}$ at $34{ }^{\circ} \mathrm{C}$ for 1 hour, and then incubated in room temperature. The solutions were bubbled with $95 \% \mathrm{O}_{2}$ and $95 \% \mathrm{CO}_{2}$. 
To visualize D2dr expressing neurons, Shank3 TG mice were crossed with D2-Cre and Ai9 (JAX Stock No. 007909). This resulted in expression of tdTomato in D2dr expressing neurons. Whole-cell recording was made from medium spiny neurons (MSN) with or without fluorescence in the dorsal stratum by using a patch-clamp amplifier (MultiClamp 700B, Molecular Devices, Union City, CA) under infrared differential interference contrast optics (Olympus, BX51WI) with X-cite 120LED boost high-power LED illumination system (Excelitas Canada Inc). Microelectrodes were made from borosilicate glass capillaries and had a resistance of 3-5 M $\Omega$. Data was acquired with a digitizer (DigiData 1440A, Molecular Devices). The software pClamp 10 (Molecular Devices) and Minianalysis 6.0.3 (Synaptosoft Inc, Decatur, GA) were used for data analysis. Miniature EPSCs (mEPSCs, holding at $-70 \mathrm{mV}$ ) were recorded in voltage-clamp mode in the presence of $100 \mu \mathrm{M}$ picrotoxin, $50 \mu \mathrm{M}$ D-2-amino-5-phosphonopentanoic acid (AP5), $10 \mu \mathrm{M}$ D-serine and $1 \mu \mathrm{M}$ Tetrodotoxin (TTX). The intrapipette solution contained (in $\mathrm{mM}$ ) 130 cesium methanesulfonate, $10 \mathrm{CsCl}, 5 \mathrm{QX}-314,10 \mathrm{HEPES}, 0.2 \mathrm{EGTA}, 2 \mathrm{MgCl}_{2}, 4 \mathrm{MgATP}, 0.3 \mathrm{Na}_{2} \mathrm{GTP}$, and $10 \mathrm{Na}_{2}$-phosphocreatine (pH 7.2 with $\mathrm{CsOH}$ ). Signals were filtered at $2 \mathrm{KHz}$ and sampled at $10 \mathrm{KHz}$. Data were discarded when the change in the series resistance was above $20 \%$ during the course of the experiment. The recording was performed at $25( \pm 1){ }^{\circ} \mathrm{C}$ with the help of an automatic temperature controller (Warner Instruments, Hamden, CT).

\section{BAC recombineering}

LoxP sites were introduced into a bacterial artificial chromosome (BAC) containing mus musculus Shank3 at its $5^{\prime}$ and $3^{\prime}$ ends outside of the coding region using the method originally described in Warning et $\mathrm{al}^{30}$. and as modified in Han et $\mathrm{al}^{4}$. The modified RP23-278D8 BAC clone containing an EGFP-tag at the first codon of Shank3 underwent iterative modification to introduce first the 3' LoxP site then the 5' LoxP site. The modified BAC clone was digested with Notl/Swal and a $75 \mathrm{~kb}$ fragment was purified following pulse field gel electrophoresis. The DNA fragment was injected into FVB/N embryos as previously described to generate founder mice ${ }^{4}$.

\section{Transmission Electron microscopy}

Mice were collected, weighed and sedated. Starting the perfusion, the vascular system was flushed with normal saline before perfusion with the buffered fixative. The striatum was dissected and sliced into $1 \mathrm{~mm}$ thick slices. Tissue was placed into a scintillator vial with buffered fixative and placed on a rotator for three days. The tissue was processed inside a Ted Pella Bio Wave Vacuum Microwave. Samples were fixed again, followed by $3 x$ sodium cacodylate buffer rinses, post-fixed with $1 \%$ buffered osmium tetroxide, and followed again with 3 millipore water rinses. Ethanol concentrations from $25-100 \%$ were used as the initial dehydration series, followed with propylene oxide as a final dehydrant. Samples were gradually infiltrated with 3 propylene oxide and Embed 812 resin graded ratios into 3 changes of pure resin under vacuum. Samples were allowed to infiltrate in pure resin overnight on a rotator. The samples were embedded into regular Beem capsules and cured in the oven at $62^{\circ} \mathrm{C}$ for 5 days. The polymerized samples were sectioned at $50 \mathrm{~nm}$. Grids were then stained with $1 \%$ uranyl acetate for ten minutes followed by $2.5 \%$ lead citrate for two minutes one day before TEM examination. Grids were viewed in a 
JEOL 1400 plus transmission electron microscope at 80kV. TEM images were captured using a an AMT XR-16 mid-mount 16 mega-pixel digital camera.

\section{Golgi staining of striatal neurons}

Mice were deeply anesthetized then rapidly decapitated. Brains were carefully dissected. Golgi-Cox impregnation was performed as per the rapid golgistain kit instructions (FD NeuroTechnologies, PK401). Coronal sections of 50uM thickness were cut and mounted on slides. Golgi staining was visualized in bright field mode on a Nikon spinning disk confocal microscope. Spines were counted in Image $\mathrm{J}$ by an individual blind to genotype.

\section{In vivo dopamine microdialysis and sample analysis}

Shank3 TG mice and their control littermates were anesthetized with isoflurane (1-3\%) and placed in a stereotaxic apparatus. Unilateral microdialysis cannulae (CMA Microdialysis, Stockholm, Sweden) were implanted vertically above the striatum (anterior +0.6 ; lateral +1.9 ; ventral $-1.8 \mathrm{~mm}$ from bregma, according to the coordinates of Paxinos and Franklin, 2004). Two anchor screws (PlasticsOne) were placed behind the cannula and then fixed to the skull with dental cement (Lang Dental). Buprenorphine $(0.03 \mathrm{mg} / \mathrm{kg}$, ip) was injected for pain relief. Three to four days following surgery, the guide cannulae were removed under light sedation with isoflurane and microdialysis probes were inserted (CMA7/2 mm, CMA Microdialysis). Awake, behaving mice were habituated to the microdialysis environment for 2-3 $\mathrm{h}$ while the probes were perfused with $0.25 \mathrm{mM}$ ascorbic acid in artificial cerebrospinal fluid (aCSF; $\left.127.6 \mathrm{mM} \mathrm{NaCl}, 4.02 \mathrm{mM} \mathrm{KCl}, 750 \mu \mathrm{M} \mathrm{NaH}_{2} \mathrm{PO}_{4}, 2.1 \mathrm{mM} \mathrm{Na}_{2} \mathrm{HPO}_{4}, 2.00 \mathrm{mM} \mathrm{MgCl}_{2}, 1.71 \mathrm{mM} \mathrm{CaCl}_{2} ; \mathrm{pH} 7.4\right)$ at a constant rate of $2 \mu \mathrm{L} / \mathrm{min}$ for the duration of the experiment. Ascorbic acid supplementation of the aCSF was used to prevent degradation of dopamine. In order to determine basal levels of dopamine, three dialysate samples were collected every $20 \mathrm{~min}$ for one $\mathrm{h}$ following habituation. Following baseline, amphetamine ( $2 \mathrm{mg} / \mathrm{kg}$, intraperitoneally) was administered and samples were collected every 20 min for two hours. Following the conclusion of the experiment, mice were killed, brains removed and post-fixed in $4 \%$ paraformaldehyde in $0.1 \mathrm{M}$ PBS for at least 5 days. Brains were then sectioned using a sliding microtome and relevant sections were cresyl violet stained for verification of probe placement. All animals had correct probe placement.

All samples were analyzed for dopamine using HPLC-ED (HTEC-500 with Autosampler INSIGHT, Amuza, Inc., Japan) with separation using a column (PP-ODS2, Amuza) and analytes detected with a working graphite electrode (WE-3G, Amuza) and an Ag/ AgCl reference electrode (+400 mV). Mobile phase consisted of $1.5 \%$ methanol, $100 \mathrm{mM}$ phosphate buffer, $500 \mathrm{mg} / \mathrm{L}$ sodium 1-decanesulfonate, and 50 $\mathrm{mg} / \mathrm{L}$ of EDTA-Na2 ( $\mathrm{pH}$ 5.4). A standard curve of known concentrations of dopamine from $10^{-6}$ to $10^{-9} \mathrm{M}$ was established. Envision Chromatography Suite (Amuza) was used to acquire and analyze chromatographic data based on this standard curve.

\section{MRI analysis}


Fixed brains for MRI were prepared as described in Tyszka et al ${ }^{31}$. Briefly, the mice were transcardially perfused with heparinized phosphate buffered saline (PBS) followed by fixation with $4 \%$ paraformaldehyde (PFA). Post perfusion, the head was removed. The skin, muscle, ears, nose tip, and lower jaw were removed to expose the skull. The head was fixed overnight in $4 \%$ PFA at $4^{\circ} \mathrm{C}$. The head was then transferred to $40 \mathrm{ml}$ of $0.01 \%$ sodium azide in PBS and rocked for 7 days at $4^{\circ} \mathrm{C}$. The head was transferred to a solution of $5 \mathrm{mM}$ gadopentate dimeglumine (Bayer HealthCare Pharmaceuticals Inc., Wayne $\mathrm{NJ}$ ) and $0.01 \%$ sodium azide in PBS and rocked for $21-24$ days at $4^{\circ} \mathrm{C}$. Incubation with gadopentate dimeglumine improved the signal-to-noise ratio. Prior to imaging, the head was equilibrated to room temperature for 6-8 hours.

For DTI, mice brains were scanned overnight using 20 gradient directions and one unweighted, anatomical scan. All scans were acquired on a 9.4 T Bruker Avance Biospec Spectrometer, 21-cm bore horizontal scanner with a $35 \mathrm{~mm}$ volume resonator (Bruker BioSpin, Billerica, MA) with Paravision 5.1 software (Bruker Biospin, Billerica, MA). The 3D DTI scan parameters were as follows: Spin echo, b-value $=0$ and $1000 \mathrm{~s} / \mathrm{mm}^{2}, 20$ diffusion directions with one non-diffusion weighted image, $\mathrm{TR}=500 \mathrm{~ms}, \mathrm{TE}=$ $14.8 \mathrm{~ms}, \mathrm{FOV}=1.5 \times 1.0 \times 2.0 \mathrm{~cm}$, matrix $=128 \times 96 \times 96, \mathrm{NEX}=1, \delta=3 \mathrm{~ms}, \Delta=7 \mathrm{~ms}$.

The image volumes were processed using DTI studio ${ }^{32}$ and ROleditor (www.mristudio.org). The mean diffusion-weighted image volumes were skull stripped to generate a brain mask using the ROleditor function. Tensor calculations were done in DTI studio, and used to generate fractional anisotropy (FA) image volumes as well as volumes for radial diffusivity, and color maps. The unweighted anatomical map (B0) and the FA maps were used for alignment of the brains with a P60 mouse brain map. The P60 template maps were from the Johns Hopkins website (http://cmrm.med.jhmi.edu/) and included a segmentation map of 58 distinct brain regions. The alignment was carried out using the DiffeoMap software $^{33,34}$ in two steps. After upsampling the subject maps $(128 \times 96 \times 96$ voxels; $15 \times 10 \times 20 \mathrm{~mm}$ field of view) to match the P60 template map resolution ( $240 \times 160 \times 320$ voxels; same field of view; 0.0625 isotropic voxel dimensions), an initial rigid alignment using the AIR algorithm ${ }^{35}$ in DiffeoMap was carried out on the B0 maps to generate a coarse alignment of the two brains. This was followed by large deformation diffeomorphic metric mapping (LDDMM) utilizing both B0 and FA maps for alignment, as carried out on the Johns Hopkins server. The alignments were then applied to the tensor and the various maps (FA, colormap, B0, radial diffusivity), to yield aligned brain volumes. The inverse alignment transformation was applied to the template segmentation map, and then downsampled to match the original subject resolution. This generated segmentation maps for the subject brains in their original scanned orientation and resolution. Additional segmentation maps for each hemisphere and for the brain injury regions were drawn and transformed to have both template and subject space versions. Fiber tracking was carried out using DTI studio to quantitate fiber number and fiber length through specific brain regions (e.g. the thalamus). Amira (version 5.6; FEI) was utilized for presentation-quality visualization of FA maps and fiber tracking data.

\section{b-arrestin biased D2 dopamine receptor antagonists}


b-arrestin biased D2 dopamine receptor antagonists were developed as previously described ${ }^{15}$. Briefly, a cell based screen was performed in cultured cells expressing human D2 dopamine receptors and human b-arrestin 2. Compounds which inhibited b-arrestin activity by $>50 \%$ were then tested for alteration of cyclic AMP (CAMP) abundance to identify those compounds which had minimal impact on cAMP production. Compounds were then tested for off-target effects on other G-protein coupled receptors (GPCRs). Final compounds were then tested for ability to cross the blood brain barrier and displace radiolabeled $\left[{ }^{11} \mathrm{C}\right]$-Raclopride.

\section{Behavioral analysis}

Mice were pre-treated with BRD4018, BRD5814 or their respective vehicle controls by intraperitoneal injection. BRD4018 was initially dissolved in 100\% dimethyl sulfoxide (DMSO) then polyethylene glycol 400 (PEG400) and saline were added such that the final vehicle was 10\% DMSO/45\% PEG400/45\% saline. Each dose for BRD4018 was $10 \mathrm{mg} / \mathrm{kg}$. BRD5814 was dissolved in 10\%DMSO/1\% Tween 80/89\% saline. Mice were injected with $10 \mathrm{mg} / \mathrm{kg}$ (BRD4018) or 10 mg/ $\mathrm{kg}$ (BRD5814) in the morning and afternoon the day prior and in the morning one hour prior to behavioral analysis. The injection volumes for all compounds was $10 \mathrm{ml} / \mathrm{kg}$. All injections were performed by an individual blind to whether they were injected with drug or vehicle. All behavior was performed by an experimenter blind to genotype.

Open field assay - Open field assay was performed as previously described ${ }^{4}$. Mice were habituated to the behavior room for 30 minutes with $600 \mathrm{~lx}$ white light and $60 \mathrm{~dB}$ white noise. Mice were then placed in a Plexiglass chamber $(40 \times 40 \times 30 \mathrm{~cm})$ and activity measured by photobeam breaks in the $\mathrm{X}, \mathrm{Y}$ and $\mathrm{Z}$ axes (Accuscan). After measuring baseline activity for 30 minutes, mice were injected with $2 \mathrm{mg} / \mathrm{kg}$ amphetamine dissolved in normal saline and activity was again measured for one hour. Data analysis was performed with Fusion software.

Acoustic startle response and prepulse inhibition- Mice were allowed to habituate to the test chamber (San Diego Instruments) for 5 minutes in the presence of $70 \mathrm{~dB}$ white noise. The test consisted of eight trial types (no stimulus, $40 \mathrm{~ms}$ if $120 \mathrm{~dB}$ startle stimulus or three different prepulse sounds ( $20 \mathrm{~ms}$ of 74,78 or $82 \mathrm{~dB}$ ) either alone or $100 \mathrm{~ms}$ prior to the startle stimulus) which were presented in a pseudorandom order. The interval between each stimulus was 10 to 20 s.

Tail suspension testing - Mice were allowed to habituate to the testing room for 30 minutes with $6001 x$ white light and $60 \mathrm{~dB}$ white noise. Mice were then suspended by their tails for seven minutes and movements were recorded by ANY-maze software.

\section{Descriptions of clinical phenotypes of human subjects with SHANK3 duplications}

This study was reviewed and approved by the Baylor College of Medicine Institutional Review Board for human studies. Written informed consent was obtained from the legal guardians and all human research was performed in accordance with guidelines and regulations of the Baylor College of Medicine Institutional Review Board and the Declaration of Helsinki. All information was de-identified. 


\section{Protein interaction network and gene co-expression analyses}

The protein interaction network consisting of interactions between human proteins and those between mouse proteins was downloaded from the BioGRID database (https://thebiogrid.org/) ${ }^{19}$. For the calculation of shortest path length, we used all the proteins in the largest connected component of the protein interaction network as the background proteins. The shortest path length from a protein to D2dr/D1dr is defined as the smallest number of protein interactions linking the protein to D2dr/D1dr in the protein interaction network.

Single-cell RNA sequencing data of mouse striatum ${ }^{21}$ were downloaded from the NCBI Gene Expression Omnibus under the accession number GSE82187. The counts of each cell were normalized to counts per million (CPM), in which CPM is the count of each gene divided by the total count of the cell and multiplied by one million. For each of D2 and D1 neuron types, genes were considered to be expressed in that type if the expression level was $>0$ in at least $10 \%$ of cells for that type. Gene co-expression networks were constructed as previously described ${ }^{20}$. For each of D2 and D1 neuron types, we used genes expressed in the neuron type as background genes. We first computed the pairwise Spearman's rank correlation coefficients between background genes and sorted all the pairwise Spearman's correlation coefficients in descending order. Then, we determined the correlation threshold for the top $1 \%$ highest pairwise Spearman's correlation coefficients, and used the correlation threshold to construct a gene co-expression network. To calculate the correlation with Shank3 interactors in D2 neurons, genes expressed in D2 neurons were used as background genes. For any Shank3 interactor in the background gene set, the correlation with Shank3 interactors for the gene was defined as the average Spearman's correlation coefficients between the gene and the other Shank3 interactors. For any non-Shank3 interactor in the background gene set, the correlation with Shank3 interactors for the gene was defined as the average Spearman's correlation coefficients between the gene and Shank3 interactors. Based on the correlation with Shank3 interactors for any background gene, we obtained the distribution of correlations with Shank3 interactors for all the background genes and calculated the percentile of the correlation with Shank3 interactors for D2dr. A similar analysis was performed to calculate the correlation with Shank3 interactors in D1 neurons using genes expressed in D1 neurons as background genes.

\section{Declarations}

\section{ACKNOWLEDGEMENTS}

This work was funded by a grant from the National Institute of Neurological Disorders and Stroke (NINDS) to JLH (NS091381) and the Philip R. Dodge Young Investigator Award from the Child Neurology Society to JLH. JLH is also generously funded by the Joan and Stanford Alexander Endowed Chair for Neuropsychiatric Genetics as well as Mr. Charif Souki. Research reported in this publication was supported by the Eunice Kennedy Shriver National Institute of Child Health \& Human Development of the National Institutes of Health under Award Number P50HD103555 for use of the Animal Phenotyping \& Preclinical Endpoints and Cell and Tissue Pathogenesis Core 
subcore facilities. The content is solely the responsibility of the authors and does not necessarily represent the official views of the National Institutes of Health. We are indebted to Emily Shaffer for critical editing of the text, Lita Duraine and Hugo Bellen for assistance with electron microscopy and G. Schuster for Shank3 BAC injections.

\section{AUTHOR CONTRIBUTIONS}

J.L.H. conceived the study, conducted and managed all experiments, analyzed and interpreted the data, drafted and revised the manuscript. H.Y.Z interpreted the data and revised the manuscript. K.P. and Z.L performed bioinformatic analyses and revised the manuscript. M.W., K.H., K.L.E.J, E.H, S.E.P, R.G.P, D.S., F.W. collected and analyzed data and revised the manuscript. W.W., T.E., B.V., M.L, Q.X., J.G. collected and analyzed the data.

\section{COMPETING INTERESTS}

The authors declare no competing interests.

\section{References}

1 Bai, D. et al. Association of Genetic and Environmental Factors With Autism in a 5-Country Cohort. JAMA Psychiatry 76, 1035-1043, doi:10.1001/jamapsychiatry.2019.1411 (2019).

2 Pearlson, G. D. Etiologic, phenomenologic, and endophenotypic overlap of schizophrenia and bipolar disorder. Annu Rev Clin Psychol 11, 251-281, doi:10.1146/annurev-clinpsy-032814-112915 (2015).

3 Satterstrom, F. K. et al. Large-Scale Exome Sequencing Study Implicates Both Developmental and Functional Changes in the Neurobiology of Autism. Cell 180, 568-584 e523, doi:10.1016/j.cell.2019.12.036 (2020).

4 Han, K. et al. SHANK3 overexpression causes manic-like behaviour with unique pharmacogenetic properties. Nature 503, 72-77, doi:10.1038/nature12630 (2013).

5 Johannessen, M., Haugen, I. B., Bakken, T. L. \& Braaten, O. A 22q13.33 duplication harbouring the SHANK3 gene: does it cause neuropsychiatric disorders? BMJ Case Rep 12, doi:10.1136/bcr-2018228258 (2019).

6 Ujfalusi, A. et al. 22q13 Microduplication Syndrome in Siblings with Mild Clinical Phenotype: Broadening the Clinical and Behavioral Spectrum. Mol Syndromol 11, 146-152, doi:10.1159/000507103 (2020).

7 Peca, J. et al. Shank3 mutant mice display autistic-like behaviours and striatal dysfunction. Nature 472, 437-442, doi:10.1038/nature09965 (2011). 
8 Mclntyre, R. S. et al. Bipolar disorders. Lancet 396, 1841-1856, doi:10.1016/S0140-6736(20)315440 (2020).

9 Borgelt, L. et al. Neurophysiological effects of multiple mood episodes in bipolar disorder. Bipolar Disord 21, 503-513, doi:10.1111/bdi.12782 (2019).

10 Shi, J. et al. Resting-state functional connectivity of neural circuits associated with primary and secondary rewards in patients with bipolar disorder. Soc Cogn Affect Neurosci 15, 755-763, doi:10.1093/scan/nsaa100 (2020).

11 Kim, W., Won, S. Y. \& Yoon, B. J. CRMP2 mediates GSK3beta actions in the striatum on regulating neuronal structure and mania-like behavior. J Affect Disord 245, 1079-1088, doi:10.1016/j.jad.2018.10.371 (2019).

12 Madisen, L. et al. A robust and high-throughput Cre reporting and characterization system for the whole mouse brain. Nat Neurosci 13, 133-140, doi:10.1038/nn.2467 (2010).

13 Jones, S. R. et al. Profound neuronal plasticity in response to inactivation of the dopamine transporter. Proc Natl Acad Sci U S A 95, 4029-4034, doi:10.1073/pnas.95.7.4029 (1998).

14 Ralph, R. J., Paulus, M. P., Fumagalli, F., Caron, M. G. \& Geyer, M. A. Prepulse inhibition deficits and perseverative motor patterns in dopamine transporter knock-out mice: differential effects of D1 and D2 receptor antagonists. J Neurosci 21, 305-313 (2001).

15 Weiwer, M. et al. Functionally Biased D2R Antagonists: Targeting the beta-Arrestin Pathway to Improve Antipsychotic Treatment. ACS Chem Bio/ 13, 1038-1047, doi:10.1021/acschembio.8b00168 (2018).

16 Masri, B. et al. Antagonism of dopamine D2 receptor/beta-arrestin 2 interaction is a common property of clinically effective antipsychotics. Proc Natl Acad Sci U S A 105, 13656-13661, doi:10.1073/pnas.0803522105 (2008).

17 Allen, J. A. et al. Discovery of beta-arrestin-biased dopamine D2 ligands for probing signal transduction pathways essential for antipsychotic efficacy. Proc Natl Acad Sci U S A 108, 18488-18493, doi:10.1073/pnas.1104807108 (2011).

18 Sakai, Y. et al. Protein interactome reveals converging molecular pathways among autism disorders. Sci Transl Med 3, 86ra49, doi:10.1126/scitranslmed.3002166 (2011).

19 Stark, C. et al. BioGRID: a general repository for interaction datasets. Nucleic Acids Res 34, D535539, doi:10.1093/nar/gkj109 (2006).

20 Pang, K. et al. Coexpression enrichment analysis at the single-cell level reveals convergent defects in neural progenitor cells and their cell-type transitions in neurodevelopmental disorders. Genome Res 30, 
835-848, doi:10.1101/gr.254987.119 (2020).

21 Gokce, O. et al. Cellular Taxonomy of the Mouse Striatum as Revealed by Single-Cell RNA-Seq. Cell Rep 16, 1126-1137, doi:10.1016/j.celrep.2016.06.059 (2016).

22 Orru, G. \& Carta, M. G. Genetic Variants Involved in Bipolar Disorder, a Rough Road Ahead. Clin Pract Epidemiol Ment Health 14, 37-45, doi:10.2174/1745017901814010037 (2018).

23 Wang, W. et al. Striatopallidal dysfunction underlies repetitive behavior in Shank3-deficient model of autism. J Clin Invest 127, 1978-1990, doi:10.1172/JCl87997 (2017).

24 Jimenez-Gomez, A. et al. Phenotypic characterization of individuals with SYNGAP1 pathogenic variants reveals a potential correlation between posterior dominant rhythm and developmental progression. J Neurodev Disord 11, 18, doi:10.1186/s11689-019-9276-y (2019).

25 Vlaskamp, D. R. M. et al. SYNGAP1 encephalopathy: A distinctive generalized developmental and epileptic encephalopathy. Neurology 92, e96-e107, doi:10.1212/WNL.0000000000006729 (2019).

26 Guo, H. et al. Disruptive mutations in TANC2 define a neurodevelopmental syndrome associated with psychiatric disorders. Nat Commun 10, 4679, doi:10.1038/s41467-019-12435-8 (2019).

27 Kim, Y., Santos, R., Gage, F. H. \& Marchetto, M. C. Molecular Mechanisms of Bipolar Disorder: Progress Made and Future Challenges. Front Cell Neurosci 11, 30, doi:10.3389/fncel.2017.00030 (2017).

28 Mertens, J. et al. Differential responses to lithium in hyperexcitable neurons from patients with bipolar disorder. Nature 527, 95-99, doi:10.1038/nature15526 (2015).

29 Li, C. T., Yang, K. C. \& Lin, W. C. Glutamatergic Dysfunction and Glutamatergic Compounds for Major Psychiatric Disorders: Evidence From Clinical Neuroimaging Studies. Front Psychiatry 9, 767, doi:10.3389/fpsyt.2018.00767 (2018).

30 Warming, S., Costantino, N., Court, D. L., Jenkins, N. A. \& Copeland, N. G. Simple and highly efficient BAC recombineering using galK selection. Nucleic Acids Res 33, e36, doi:10.1093/nar/gni035 (2005).

31 Tyszka, J. M., Readhead, C., Bearer, E. L., Pautler, R. G. \& Jacobs, R. E. Statistical diffusion tensor histology reveals regional dysmyelination effects in the shiverer mouse mutant. Neuroimage 29, 10581065, doi:10.1016/j.neuroimage.2005.08.037 (2006).

32 Jiang, H., van Zijl, P. C., Kim, J., Pearlson, G. D. \& Mori, S. DtiStudio: resource program for diffusion tensor computation and fiber bundle tracking. Computer methods and programs in biomedicine 81, 106116, doi:10.1016/j.cmpb.2005.08.004 (2006). 
33 Zhang, W. et al. Landmark-referenced voxel-based analysis of diffusion tensor images of the brainstem white matter tracts: application in patients with middle cerebral artery stroke. Neuroimage 44, 906-913, doi:10.1016/j.neuroimage.2008.09.013 (2009).

34 Ceritoglu, C. et al. Multi-contrast large deformation diffeomorphic metric mapping for diffusion tensor imaging. Neuroimage 47, 618-627, doi:10.1016/j.neuroimage.2009.04.057 (2009).

35 Woods, R. P., Grafton, S. T., Watson, J. D., Sicotte, N. L. \& Mazziotta, J. C. Automated image registration: II. Intersubject validation of linear and nonlinear models. Journal of computer assisted tomography 22, 153-165 (1998).

\section{Figures}


A

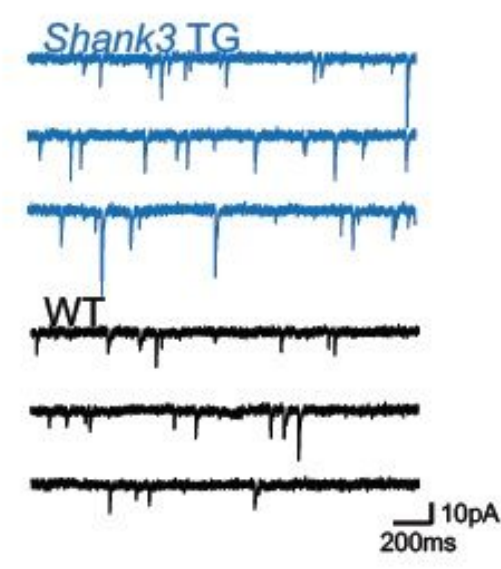

D

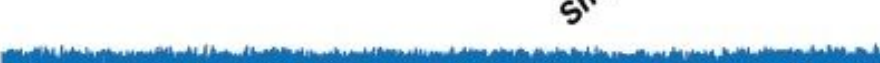

B

\section{Striatal mEPSCs}

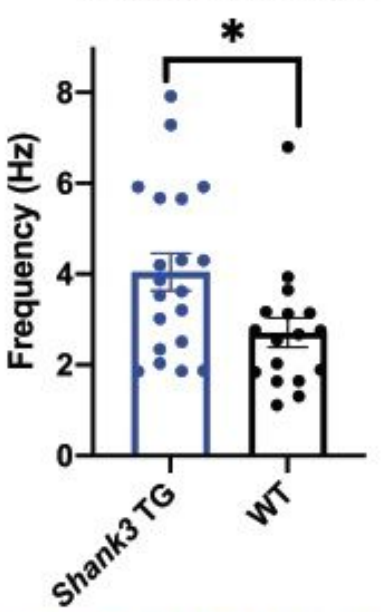

C

\section{Striatal mEPSCs}

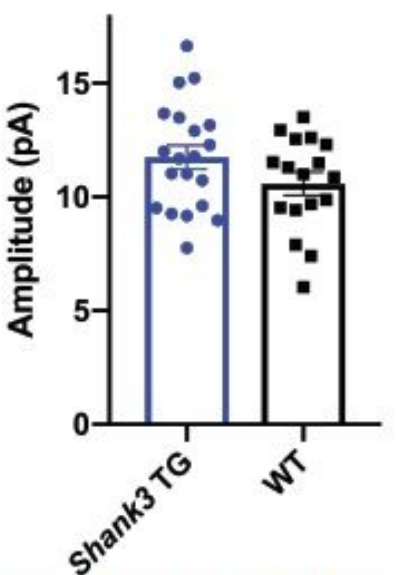

Shank3 TG-D2

WT-D2

Shank3 TG - D1
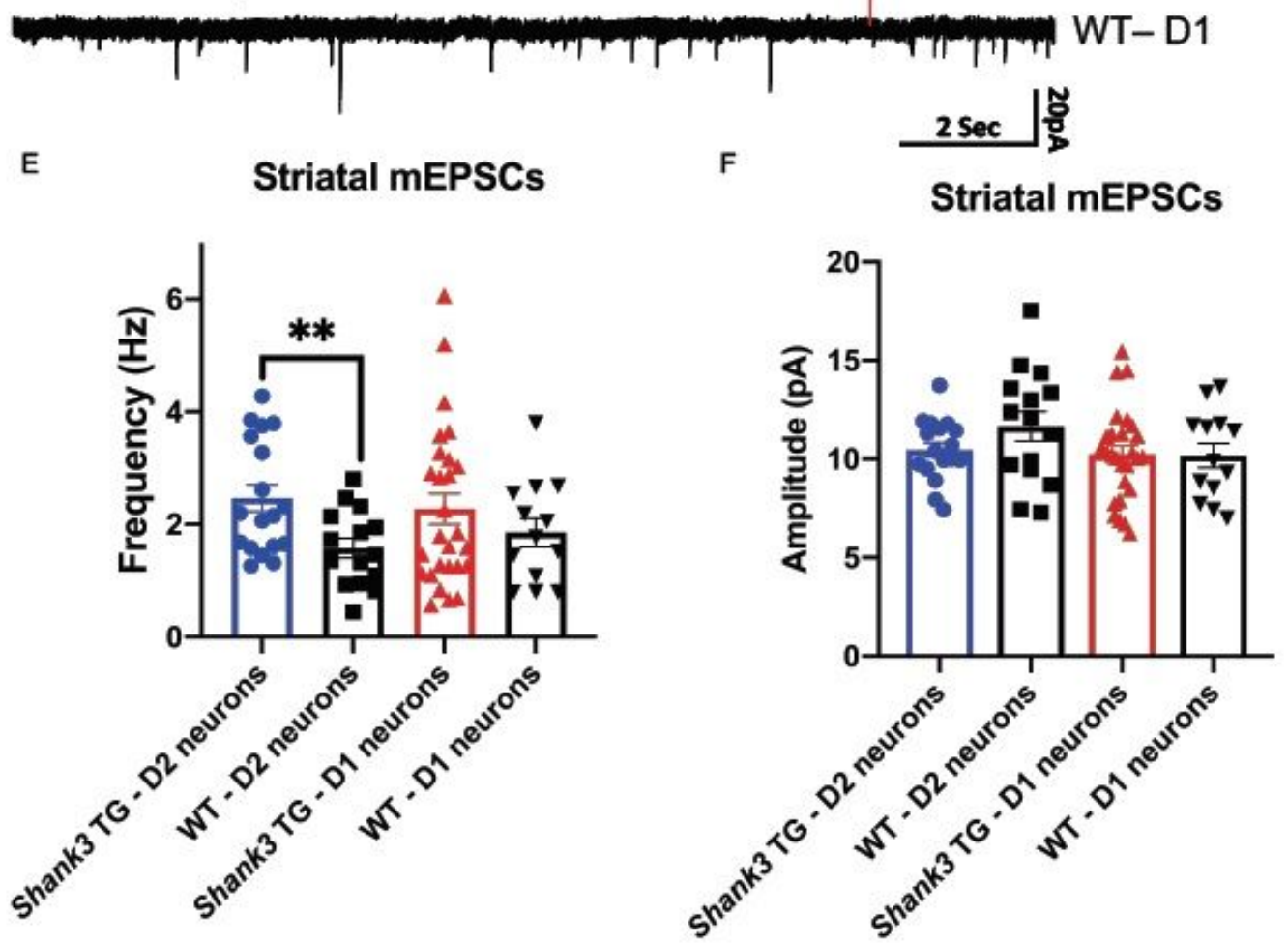

Figure 1

Neurophysiologic characterization of medium spiny neurons in Shank3 TG mice. (A) Patch-clamp miniature Excitatory Post-Synaptic Current (mEPSC) recordings from Shank3 TG mice and wild-type (WT) littermates. Cumulative data from Shank3 TG and WT mice for (B) frequency and (C) amplitude of mEPSCs of striatal medium spiny neurons. Data reveal significant increase in mEPSC frequency but normal amplitude in Shank3 TG mice. $n=21$ Shank3 TG neurons from 3 mice, $n=17$ WT neurons from 3 
mice. (D) mEPSC patch-clamp recordings from Shank3 TG D2 (blue) and D1 (red) and WT D2 (black) and D1 (black) medium spiny neurons. Cumulative data from Shank3 TG and WT mice for (E) frequency and (F) amplitude of D2 and D1 dopamine receptor expressing medium spiny neurons. Data reveal a significant increase in mEPSC frequency of only D2 neurons in Shank3 TG mice. No difference in mEPSC amplitude was noted in either D2 or D1 neurons. $n=19$ Shank3 TG D2 neurons from 3 mice, $n=15$ WT D2 neurons from 2 mice, $n=27$ Shank3 TG D1 neurons from 3 mice, $n=13$ WT D1 neurons from 2 mice. ${ }^{*} \mathrm{p}<0.05,{ }^{*} \mathrm{p}<0.01$, Student's T-test.

A
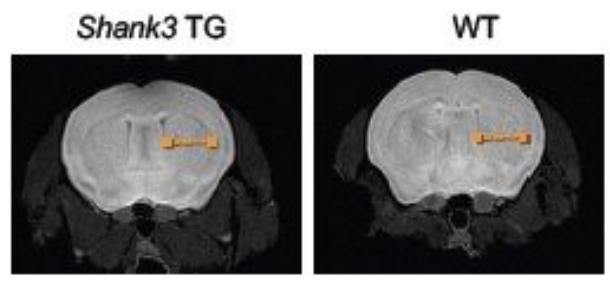

D
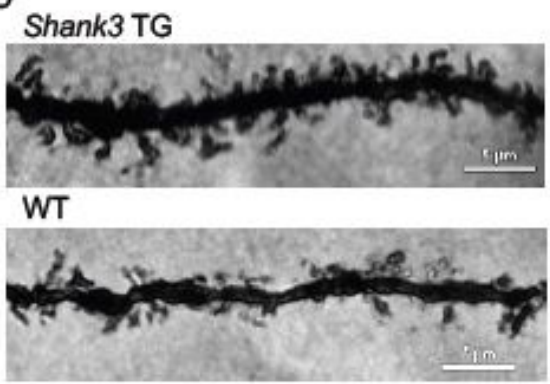

B

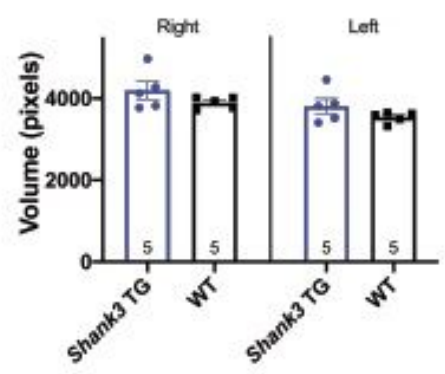

E

\section{Caudate Fractional Anisotropy}

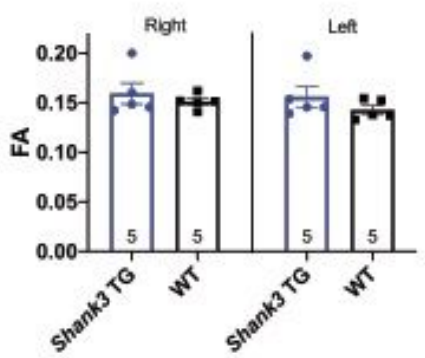

F

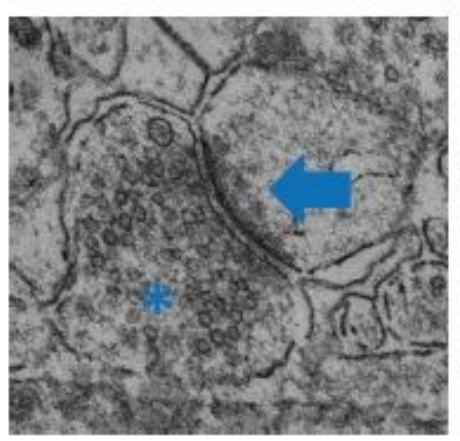

I

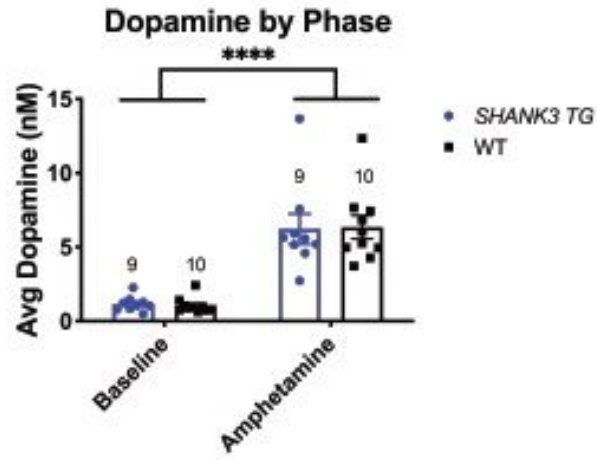

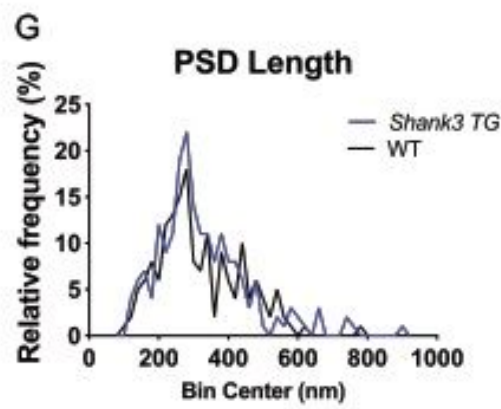

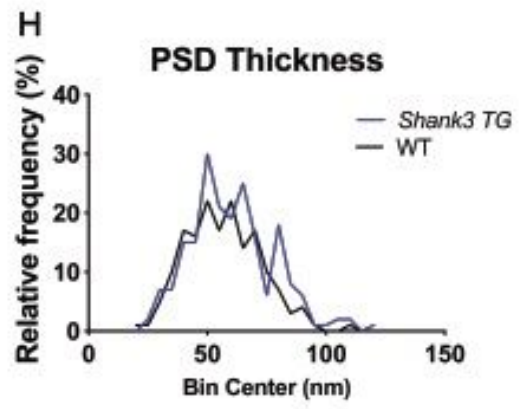


Evaluation of striatal structure and dopamine content. (A) Coronal sections of MRI imaging through the striatum of Shank3 TG and WT mice. Bar indicates location of striatum. Volume (B) and (C) fractional anisotropy of the caudate of Shank3 TG and WT mice. N=5 Shank3 TG and N=5 WT mice.

Photomicrograph of Golgi stained dendrites of Shank3 TG and WT mice of medium spiny neurons reveals significantly increased dendritic spine number (E) in Shank3 TG mice. $N=65$ Shank3 TG and N=49 WT dendrites. (F) Electron micrograph of a synapse in the striatum of a Shank3 TG mouse. No significant difference was identified in post-synaptic density $(G)$ length or $(H)$ thickness. $n=202$ Shank3 TG and $\mathrm{n}=168$ WT synapses. Dopamine efflux measured by in vivo microdialysis and high performance liquid chromatography revealed no significant difference at either baseline or post-amphetamine administration $\mathrm{N}=9$ Shank3 TG and N=10 WT mice (I). Amphetamine significantly increased extracellular dopamine in the striatum of both Shank3 TG and WT littermate mice (I and J). ${ }^{*}<<0.05$, Student's T-test.

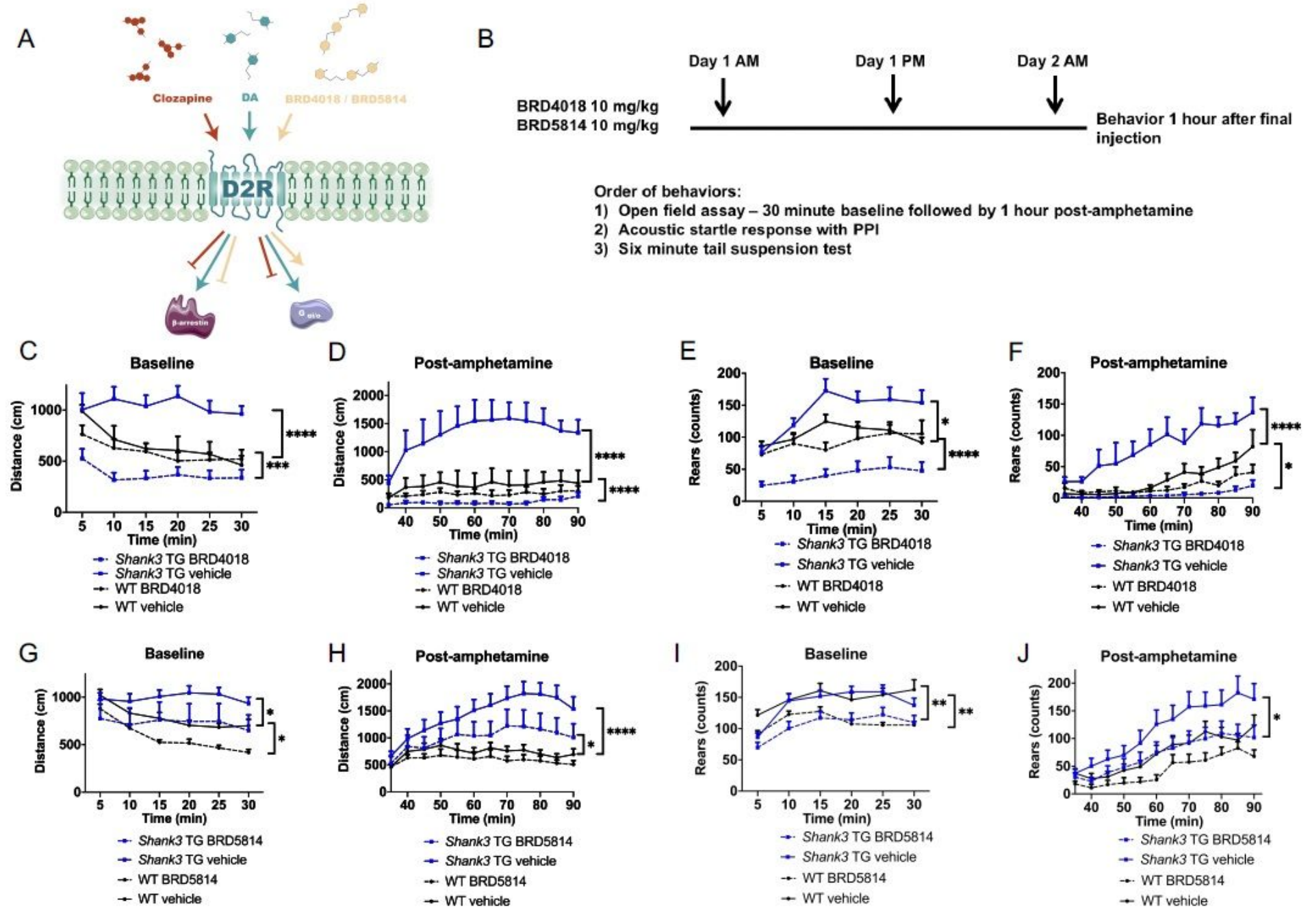

Figure 3

\-arrestin biased D2 dopamine receptor antagonists rescue baseline and amphetamine induced hyperactivity. (A) BRD4018 and BRD5814 are D2 dopamine receptor antagonists biased for inhibiting 


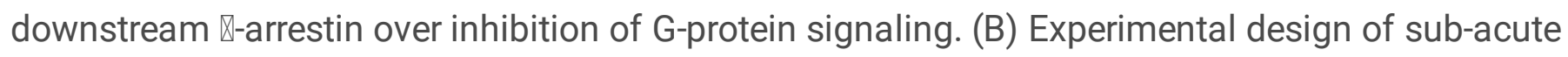
injections of BRD4018 and BRD5814 in Shank3 TG and WT mice prior to behavior analysis. BRD4018 reduced both baseline (C) and exaggerated amphetamine induced hyperactivity (D) of Shank3 TG mice; Shank3 TG BRD4018 $N=12$, Shank3 TG vehicle $N=10$, WT BRD4018 $N=11$, WT vehicle $N=12$. Rescue of increased baseline $(E)$ and amphetamine induced $(F)$ rearing in Shank3 TG mice by BRD4018; Shank3 TG BRD $4018 \mathrm{~N}=12$, Shank3 TG vehicle $\mathrm{N}=10$, WT BRD $4018 \mathrm{~N}=11$, WT vehicle $\mathrm{N}=12$. BRD5814 reduced both baseline $(G)$ and exaggerated amphetamine induced hyperactivity $(H)$ in Shank3 TG mice; Shank3 TG BRD5814 N=16, Shank3 TG vehicle $N=20, W T$ BRD5814 N=21, WT vehicle $N=17$. Rescue of increased baseline (I) and amphetamine induced (J) rearing in Shank3 TG mice by BRD5814; Shank3 TG BRD5814 $N=16$, Shank3 TG vehicle $N=20$, WT BRD5814 $N=21$, WT vehicle $N=17$. One-way nested ANOVA with

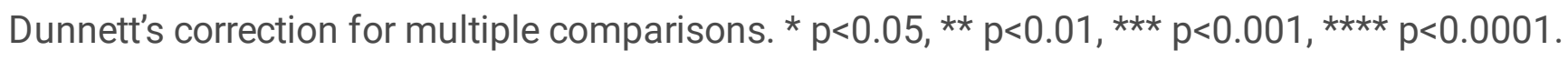




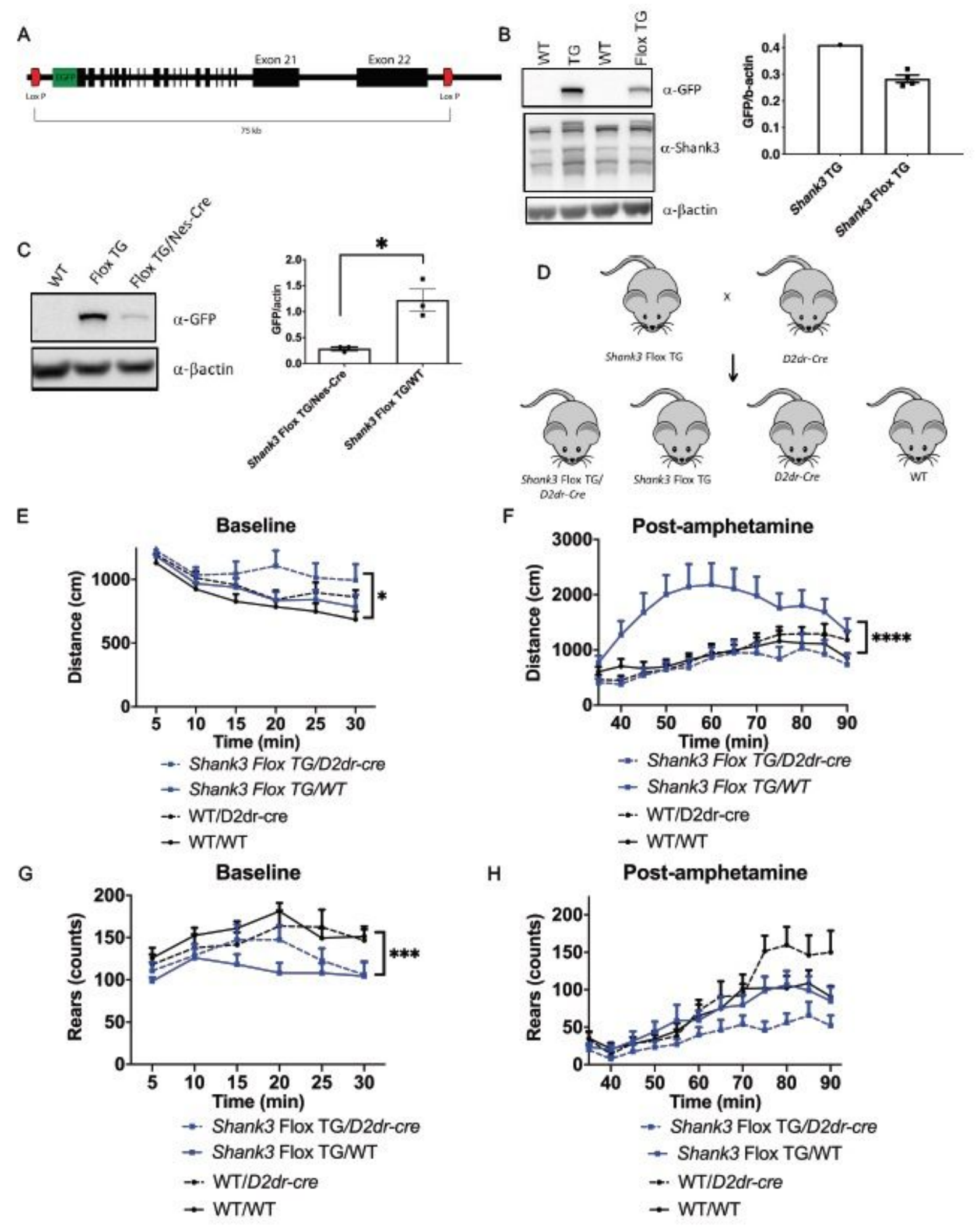

Figure 4

Rescue of exaggerated amphetamine induced hyperactivity by normalizing Shank3 in D2 dopamine receptor expressing cells. (A) Diagram of engineered mouse Bacterial Artificial Chromosome (BAC) with LoxP sites introduced at 5' and 3' ends of the Shank3 gene (Shank3 Flox TG line). (B) Western blot of protein from cerebrum from Shank3 TG, Shank3 Flox TG and WT mice. By blotting with anti-GFP antibody, Shank3 Flox TG mice have approximately a 35\% increase in the abundance of the long isoform 
of Shank3 compared with wild-type whereas the original Shank3 TG line has approximately a $50 \%$ increase4. (C) Shank3 Flox TG mice were mated with mice harboring a Nes-Cre transgene to generate double transgenic animals. Double transgenic animal have approximately an $80 \%$ reduction in GFPShank3 abundance compared with Shank3 Flox TG animals. (D) Mating scheme to normalize Shank3 abundance in D2 dopamine receptor expressing cells in Shank3 Flox TG/D2dr-Cre animals. Total activity by open field assay at (E) baseline and in response to (F) amphetamine. Shank3 Flox TG animals have normal baseline activity whereas the have an exaggerated amphetamine induced hyperactivity. Normalization of Shank3 in D2 neurons rescues the response to amphetamine. Rearing activity in Shank3 Flox TG mice at (G) baseline and in response to $(H)$ amphetamine. Shank3 Flox TG/D2-Cre $N=14$, Shank3 Flox TG N=17, D2-Cre N=14, WT N=19. For behavior, one-way nested ANOVA with Dunnett's

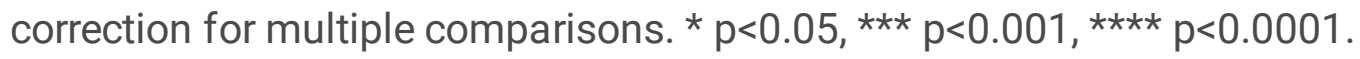




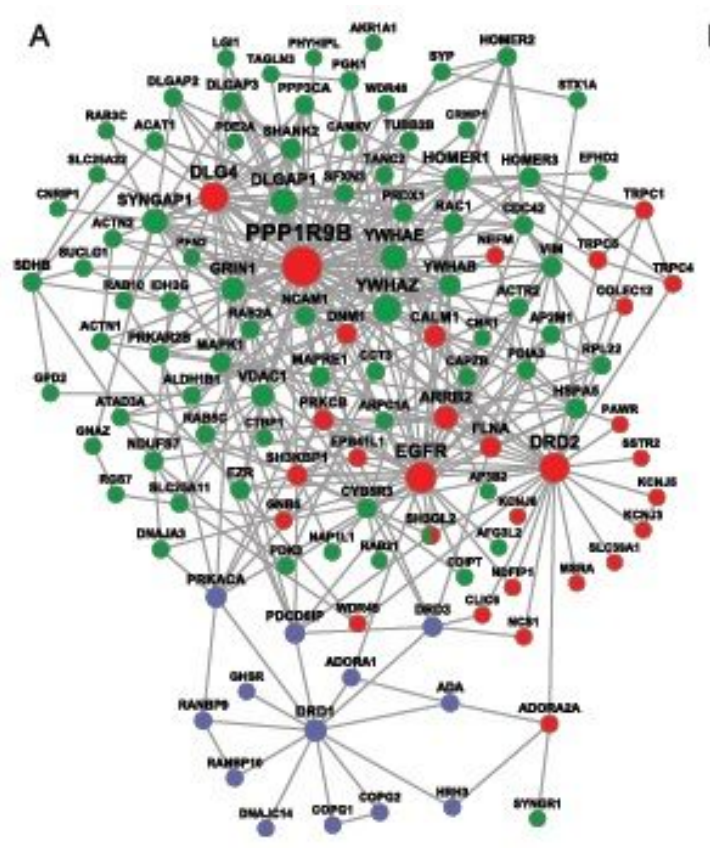

B
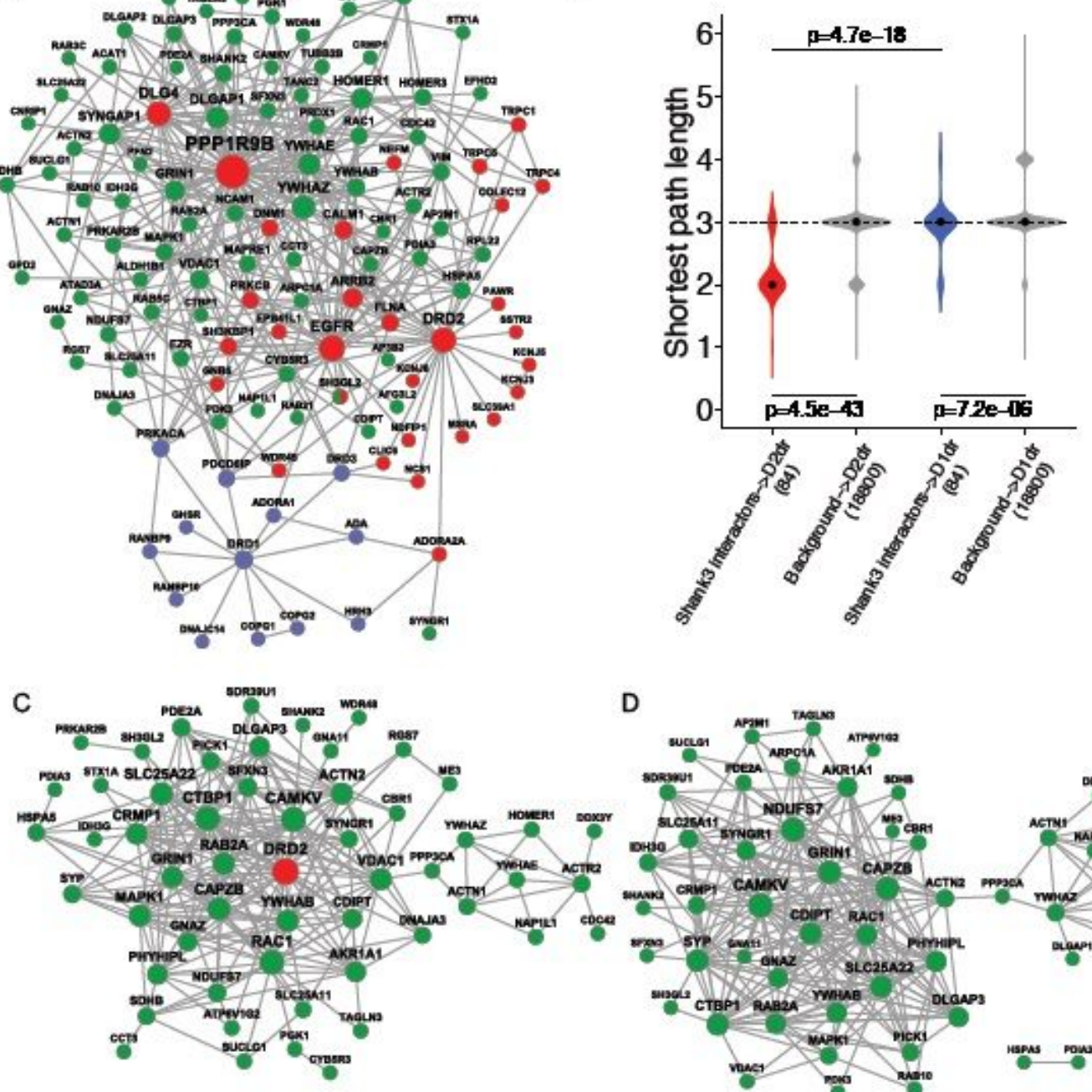

E

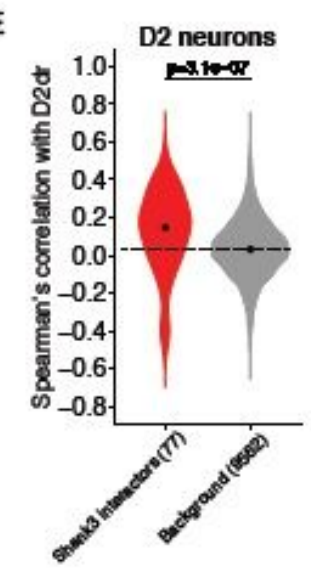

$\mathbf{F}$

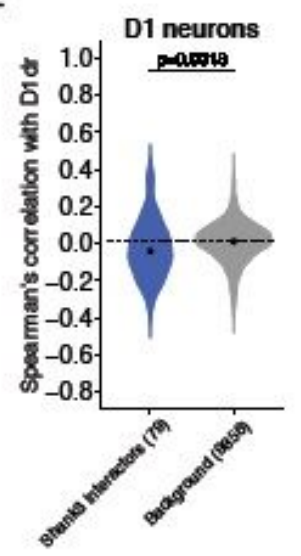

G

D
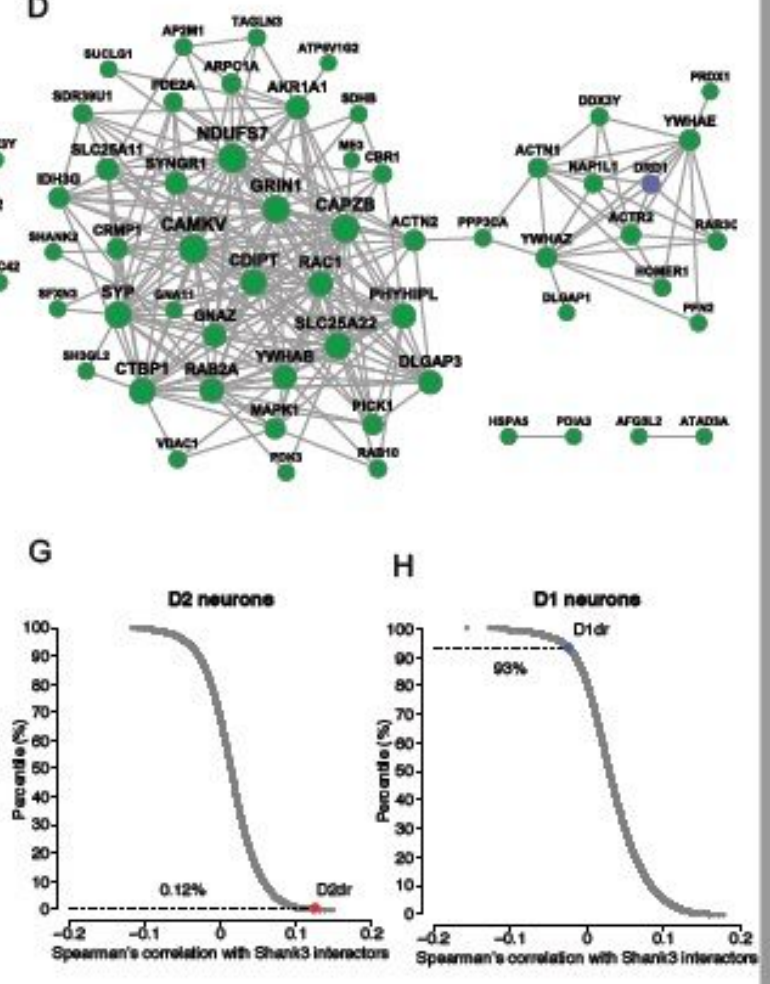

\section{Figure 5}

High-confidence Shank3 striatal interactome functions closely with D2dr interactors and co-expressed genes. (A) Protein interaction network of high-confidence Shank3 interactors, D2dr with its interactors, and D1dr with its interactors. Node size is proportional to number of protein interactions. Green, red, and blue nodes indicate Shank3 interactors, D2dr with its interactors, and D1dr with its interactors, respectively. (B) Shortest path length of Shank3 interactors and background proteins with D2dr and D1dr 
in protein interaction network demonstrates closer physical distance between Shank3 and D2dr. Violin plot shows the median value (point). P-value indicates shortest path length difference (one-sided Wilcoxon rank-sum test). Gene set size is shown in parentheses. (C) Gene co-expression network of Shank3 interactors and D2dr in D2 neurons. Node size is proportional to number of co-expressed genes. Green and red nodes indicate Shank3 interactors and D2dr, respectively. (D) Gene co-expression network of Shank3 interactors and D1dr in D1 neurons. Node size is proportional to number of co-expressed genes. Green and blue nodes indicate Shank3 interactors and D1dr, respectively. (E,F) Spearman's correlation of Shank3 interactors and background genes with (E) D2dr in D2dr-expressing neurons (D2 neurons) and (F) D1dr in D1dr-expressing neurons (D1 neurons). Violin plot shows the median value (point). P-value indicates correlation difference (one-sided Wilcoxon rank-sum test). Gene set size is shown in parentheses. (G) Scatter plot shows the distribution of Spearman's correlation with Shank3 interactors in D2 neurons for all the genes expressed in D2 neurons. Dots represent individual genes. The dashed horizontal line at $0.12 \%$ indicates the top percentile among which the correlation between Shank3 interactors and D2dr is ranked. $(\mathrm{H})$ Scatter plot shows the distribution of Spearman's correlation with Shank3 interactors in D1 neurons for all the genes expressed in D1 neurons. Dots represent individual genes. The dashed horizontal line at $93 \%$ indicates the top percentile among which the correlation between Shank3 interactors and D1dr is ranked.

\section{Supplementary Files}

This is a list of supplementary files associated with this preprint. Click to download.

- SUPPLEMENTALTABLEI.pdf

- SUPPLEMENTALTABLEII.pdf

- SUPPLEMENTALTABLEIII.pdf

- SUPPLEMENTALTABLEIV.pdf

- SUPPLEMENTALTABLEV.pdf

- SUPPLEMENTALTABLEVI.pdf

- SUPPLEMENTALFIGURE1.pdf

- SUPPLEMENTALFIGURE2.pdf

- SUPPLEMENTALFIGURE3.pdf 